\title{
Spatial Characteristics of Walking Areas for Pedestrian Navigation
}

\author{
Jianghua Zheng, Adam Winstanley \\ Dept. of Computer Science \\ National University of Ireland Maynooth \\ Maynooth, Co.Kildare, Ireland \\ Jianghua.zheng@nuim.ie
}

\author{
Zheng Pan, Seamus Coveney \\ National Centre for GeoComputation, Ireland \\ National University of Ireland Maynooth \\ Maynooth, Co.Kildare, Ireland \\ Pan.zheng@126.com
}

\begin{abstract}
We consider how to handle open walking areas in pedestrian navigation applications. Walking areas are a key type of feature which is a major difference between networks of pedestrian navigation and that of road vehicle navigation. Up to recently, little work has been carried out on these features though they are important to route planning algorithm design and path representation for pedestrian navigation. Characteristics of walking areas are classified into three types with orthogonal attributes, including the character of boundaries and entrances, concavo-convex characteristics of shapes and the presence and numbers of islands. The possible combinations are identified and general solutions proposed. Future work including the extension to 3D navigation in buildings and trials in mobile applications are proposed.
\end{abstract}

Keywords location based services, pedestrian navigation, walking area, route planning, representation, topology

\section{INTRODUCTION}

Finding one's way in unknown or not very familiar environments is a common task that people experience regularly. A number of supporting tools are available, from conventional paper maps to modern electronic navigation systems. Vehicle navigation systems have long been the focus of research and are probably the most widely and successfully used example of mobile applications. Pedestrian navigation has also been talked about for a long time. Despite the fact that many navigation solutions do include a pedestrian mode setting, none of these are really worthy of that name as they include few adaptations for the pedestrian environment [1].

Pedestrians are not constrained to the road network (for example the lanes, turn restrictions, one-way streets) unlike vehicle drivers [2], [3]. In addition there are walking areas where pedestrians can walk freely such as squares, parks and open ground. This is one of the key differences compared with the road networks used for vehicle navigation. Modeling the spatial relationship of walking areas is necessary for useful pedestrian route planning and path representation.

The eCampus project is constructing a major test bed for StratAG, the Strategic Research Cluster in Advanced Geotechnologies (www.stratag.ie) centered at National University of Ireland Maynooth. It focuses on constructing a campus information system including diverse location-based services. Pedestrian navigation both in $2 \mathrm{D}$ and $3 \mathrm{D}$ environments is one of its key functions. This paper describes some preliminary work achieved so far.

\section{A Brief OVERVIEW Of Pedestrian NAVIGATION}

Recent advances in mobile phones, positioning technologies and wireless networking infrastructures are making it possible to implement and operate large-scale pedestrian navigation. NAVITIME in Japan is probably the most successful commercial navigation service for the public. Pedestrian navigation is one of its main services. Currently (January 2007), 1.82 million people in Japan use the service, and this number is expected to exceed 2 million soon [4]. It main functions include:

1) an automatic route selection system;

2) a route-guidance interface with dynamic maps, turnby-turn instructions, progress bars, and other aids.

3) A 3D user interface was recently introduced for some areas and will be more widely available in the future.

NAVITIME has a user-friendly interface, but, since pedestrian navigation is only one of its functions, some requirements for pedestrians are not present, such as modeling the complexity of walking areas and indoor navigation.

In late 2004, China UNICOM released navigation service, Navigation Star, which has functions similar to NAVITIME [6]. It was developed on the BREW and CDMA1X platforms. It had over 100,000 users by the end of 2006. In 2007, it was upgraded with an audio-visual interface. China Mobile also provided a similar application, named Mobile Navigation, around the same time.

Nokia released the Maps 2.0 software for pedestrian navigation, offering several enhancements such as satellite imagery, "breadcrumb" traces for improved orientation and a simplified local search interface integrated into the map display [1]. Its typical platform is the Nokia 6210 Navigator phone. Due to the lack of detailed data, it also cannot provide a pedestrian navigation service indoors or in open walking areas. However, it does provide an orientation function indoors using an inbuilt e-compass.

There are also some other smaller-scale pedestrian navigation systems, mainly for demonstration or in niche areas. One main type is to aid people with disabilities. UAccess [6] is such a web-based routing tool, which provides pedestrians with the shortest feasible route with respect to one of three differing ability levels, namely, peripatetic 
(unaided mobility), aided mobility (mobility with the help of a cane, walker or crutches) and wheelchair users. It uses Dijkstra's shortest path algorithm. There are some other navigation prototypes for disabled people, such as MOBIC [7], Drishti [8], PONTES [9] and ODILIA [10].

Other systems focus on the demonstration of positioning technologies. Tetsuya [11] discusses M-CubITS pedestrian WYSIWYAS navigation using tile carpets. Okan [12] explores hybrid inertial/GPS systems to provide continuous positioning through varying environments for pedestrian navigation. G'rard [13] also demonstrates the use of microelectro-mechanical systems (MEMS) to aid GPS positioning for pedestrian navigation indoors. Masakatsu [14] described an embedded pedestrian navigation system composed of a self-contained sensor, GPS and an active Radio Frequency Identification (RFID) tag system for both indoor and outdoor pedestrian navigation. Retscher [15] summarizes 18 kinds of indoor technologies and constructed the prototype in the research project NAVIO (Pedestrian Navigation Systems in Combined Indoor/Outdoor Environments). Joy [16] and Tracy [17] argue the importance of landmarks in supporting pedestrian navigation.

Some researchers have worked on data modeling for pedestrian navigation; the standard ISO-GDF4 provides special features for this. Edgar-Philipp [18] put forward a graph-based semantic spatial model which can serve as a reference for guiding pedestrians inside buildings. Christian [2] created a graph model contrasting with that for vehicles. Zheng [3] focused on data modeling for outdoor pedestrian navigation and gave a solution for hybrid travel patterns. Others have carried out some preliminary work in $2.5 \mathrm{D} / 3 \mathrm{D}$ spatial representation for pedestrian navigation. Antti [19] gave a discussion of how representational differences in mobile 2D and 3D maps affect strategies of embodied interaction. Some have worked on pedestrian behavior analysis to improve services [20] [21].

The major difference between pedestrian and vehicle navigation lies in the accessibility rules for their networks [3]. In most cases, pedestrians have better accessibility than vehicles in urban regions, except in motorways. Algorithms for pedestrian navigation are centered on the shortest path problem as the key and basic part of network analysis. This not only refers to the shortest geographic distance, but also extends to other metrics, such as time, expenditure and line capacity.

The classic shortest path algorithm is Dijkstra's, which forms the basis for most current systems, although many use enhancements and heuristics to improve it. There are however in total 17 general algorithms for the shortest path problem. Zhan [22] compares 15, showing three of them were superior, namely TQQ (graph growth using two data queues), DKA (Dijkstra's algorithm using approximate buckets) and DKD (Dijkstras algorithm implemented with double buckets). The TQQ algorithm is based on graph growth theory and is suitable for calculating the shortest paths from one single point. The others, based on Dijkstra, are used to obtain the shortest path between two points. Due the hardware restrictions in the past, designers often paid more attention to memory requirements and usually sacrificed acceptable time to save storage space. However, now the efficiency of algorithms is more important.

Many field applications and prototypes of pedestrian navigation use the Dijkstra algorithm (or improved versions). [4] [6] [23]. Haque [24] discussed algorithms for reliable navigation and wayfinding, and presented a reliable path algorithm that minimizes the number of complex intersections with turn ambiguities between two locations along a route. Zheng [3] gave a preliminary discussion on the burning algorithm for pedestrian navigation. It provides a solution with the least intersections for pedestrians. Other researchers have worked on the shortest path algorithm using 2.5D/3D data [25] [26].

Overall, pedestrian navigation is still advancing as the hardware technology improves. There are still a few features to be fully realised, including dealing with open walking areas.

\section{WALKING AREAS AND PEDESTRIAN ROAD NETWORKS}

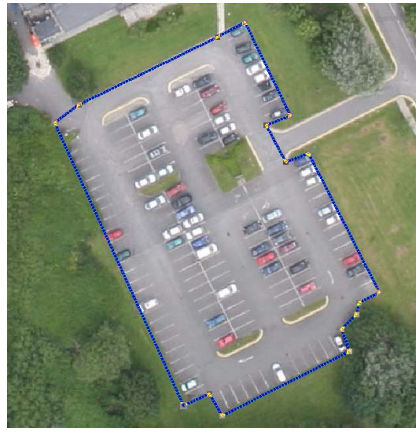

(a)

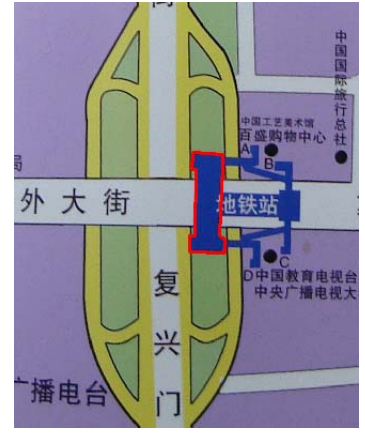

(b)

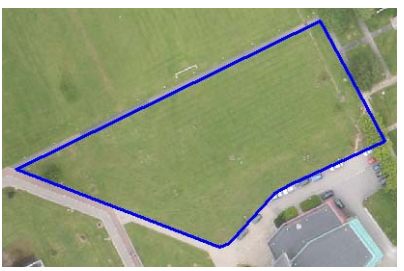

(c)

Figure 1.Example walking areas

We define a walking area as an area of free access for pedestrians with no fixed paths, such as a piece of grassland, a town square, or a courtyard. It is a common feature of pedestrian networks and distinguished it from road networks.

Figure. 1 shows typical examples of walking areas. Figure 1(a) shows a typical car park, which generally is a walking area with many entrances, (b) displays the Fuxing Gate subway station in Beijing. It is a typical walking area with a few fixed entrances. Figure 1(c) is a piece of grassland where people can enter and traverse freely from many directions. Zheng [3] has given the definition of this and related features as follows. 


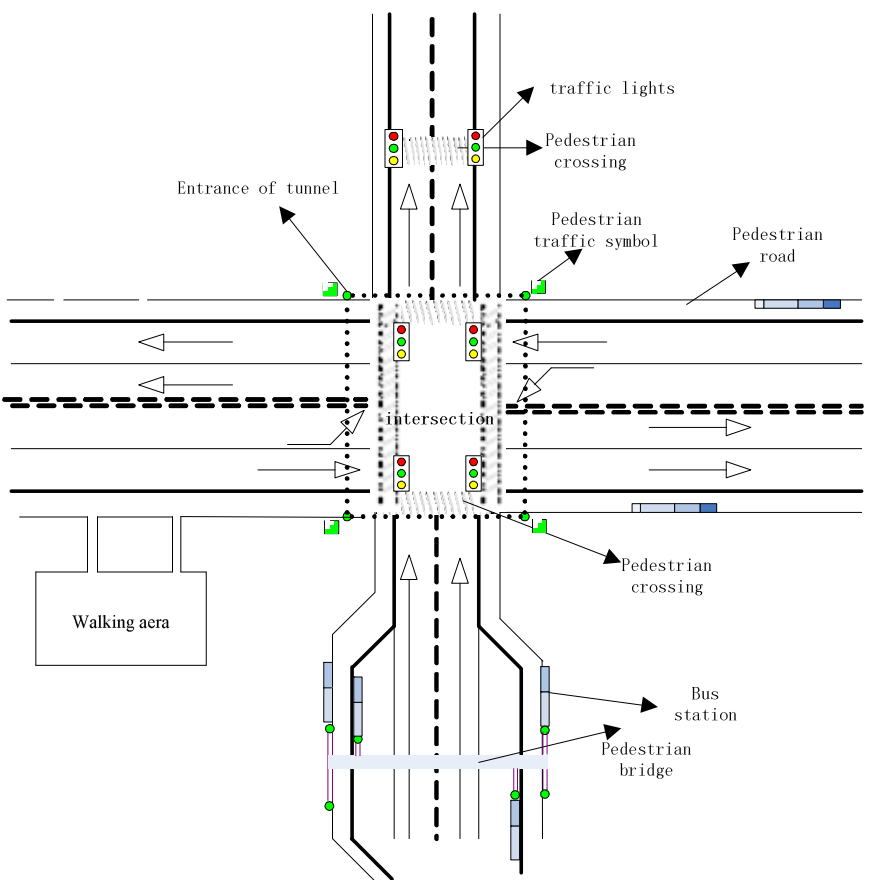

Figure 2. Pedestrian road traffic infrastructure (adapted from Xin Zhang, 2007[27], in Zheng, 2008 )

\section{A. Features in pedestrian networks}

- walking area: a region where pedestrians can walk at random, such as a square and a public transport stop area. It is represented by a polygonal feature. It usually connects to other features in the network through defined entrances/exits.

- pedestrian road link: a section of path that people can walk over, for example a crosswalk or a pedestrian overpass or underpass. It just has two ends each defined by one pedestrian road link point.

- pedestrian road link point: a point defining the end of a pedestrian road link.

- pedestrian road: It is a feature that comprises one or multiple pedestrian road links. It is used to represent a continuous named road in reality. It is used to avoid breaking road integrity by the citation of contiguous pedestrian road links in route descriptions.

- symbol point: signifies a landmark along a pedestrian road or in a walking area. It could be for example a traffic light, pedestrian traffic sign or architectural landmark.

Figure. 2 shows the major components of typical pedestrian road network. Each feature has some basic attributes, such as service status, and width. The walking area represented is an example of fixed entrance type of walking area.

\section{B. Relationships in pedestrian road networks}

Figure 3 represents the relationships between the features in pedestrian road networks

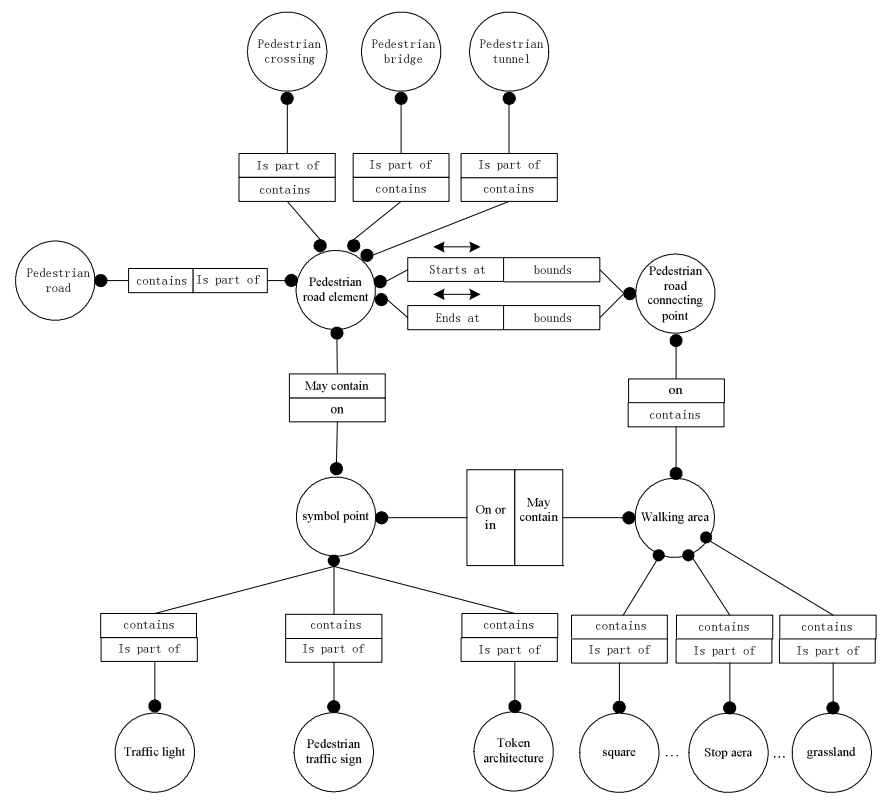

Figure.3. Relationships between features in pedestrian road networks (after Xin Zhang, 2007[27], Modified in Zheng, 2008)

\section{SPATIAL RELATIONSHIPS ANALYSING OF WALKING AREAS}

A walking area can be represented by a polygon in a logical model of pedestrian road network. The polygon represents the boundary of the area. It is made up of simple straight links and nodes. Polygons can have topological and non-topological spatial relationships. We focus on those related to path determination and path representation.

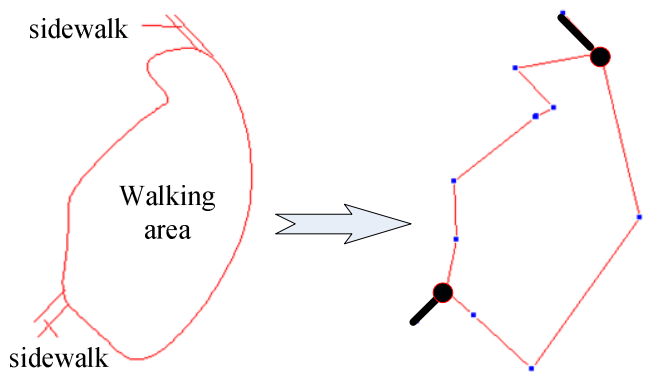

Figure 4 Abstraction of fixed entrance area

After systematical analysis and prototyping, we list the following three.

- Entrance/exit characteristics,

- concavo-convex characteristics and

- numbers and shapes of islands.

Using these characteristics, we can define various type of walking area.

- by access characteristics

1) fixed entrance

In Figure 4 black symbols stand for pedestrian road link points. In the fixed entrance situation, it also stands for the entrance/exit of the walking area. The small points are used 
to represent simple geometric elements. Dark lines are part of pedestrian road link. In this fixed entrance situation, pedestrians have no other access to the walking area except through these defined points.

2) free entrance

When the walking area is encircled by pedestrian road links, all of its boundary could be an entrance or exit.

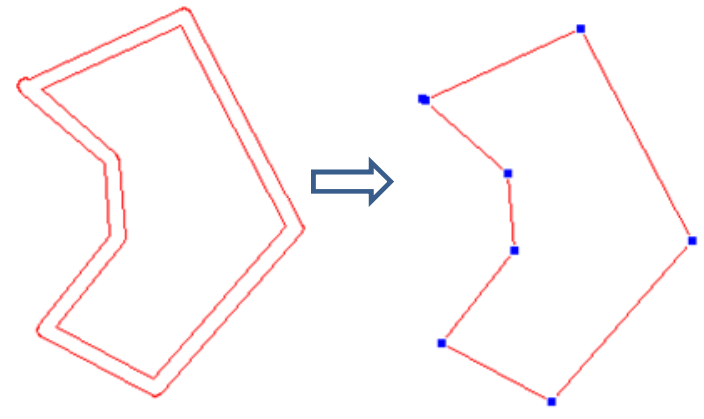

Figure.5 Free entrance areas

Figure 5 shows an example of a free entrance area. The pedestrian road link coincides with the geometry of the boundary polygon. Any point on the boundary of the walking area could be a dynamic pedestrian road link point.

\section{3) free entrance with restrictions}

Some instances of free entrance areas may have some restrictions under special situations. For example, one or multiple edges of the walking area polygon may have entrance forbidden, but the other edges are open. This is a common phenomenon in urban areas because there are often buildings or walls along one or more edges of a walking area.

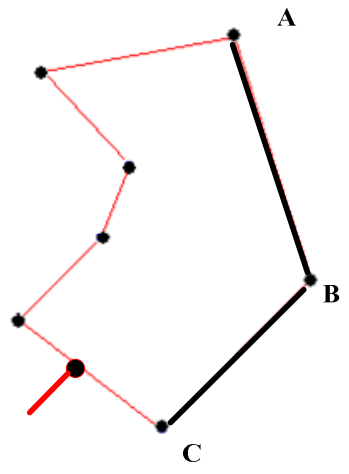

(a)

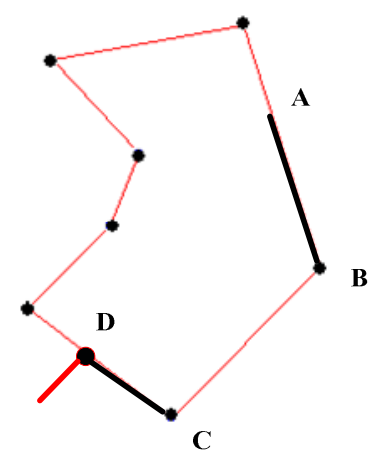

(b)
Figure 6 Free entrance area with restrictions

Edges $\mathrm{AB}$ and $\mathrm{BC}$ in Figure $6 \mathrm{a}$ represent entranceforbidden boundaries. For route planning, A, B and $\mathrm{C}$ should be ignored as accessible pedestrian link points. Figure $6 b$ represents a more complex situation.
- by concavo-convex shape characteristics

Concavo-convex characteristics of walking area shapes also impact on route planning algorithms and their resulting presentation.

1) convex shapes

Convex polygons are the simplest. It is easy to determine the optimal route in this case, because by definition any shortest path (straight line) between start and end will not intersect the boundary (Figure 7a).

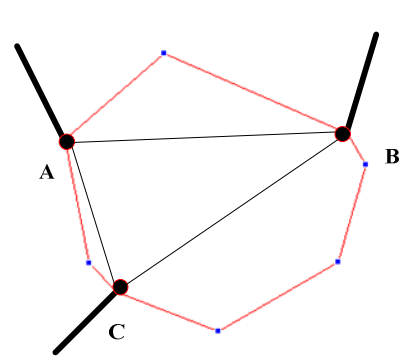

(a) convex polygon

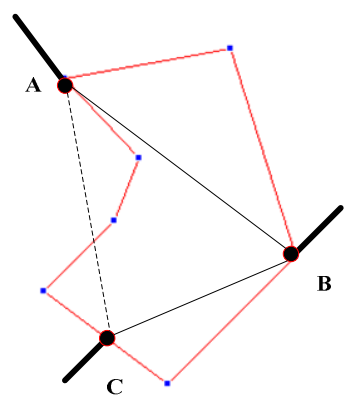

(b) concave polygon 1

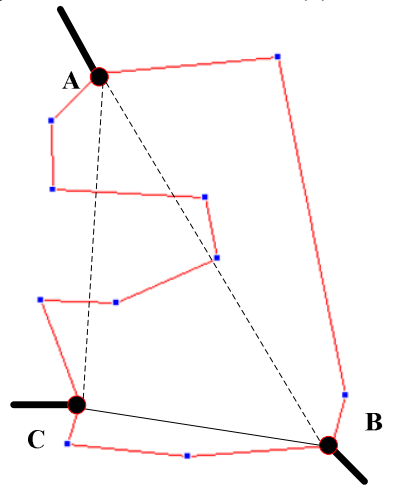

(c) concave polygon 2

Figure 7 Concavo-convex characteristics of polygons

2) concave shapes

As shown in Figure $7 \mathrm{~b}$ and $7 \mathrm{c}$, a straight-line path is not always contained in a concave area. In these cases we either have to leave and re-enter the area or plan a route around the concave segment of the boundary.

- by numbers of islands

1) without islands

This is the simplest case; all the above instances are of this type.

2) with islands

$\mathrm{A}$ and $\mathrm{B}$ in Figure 8 represent two areas pedestrians can not enter. For example there are often small borders or garden features that are out-of-bounds in a town square. In addition an island outline can be concave or convex. We must take these factors into consideration when computing the optimal route. 


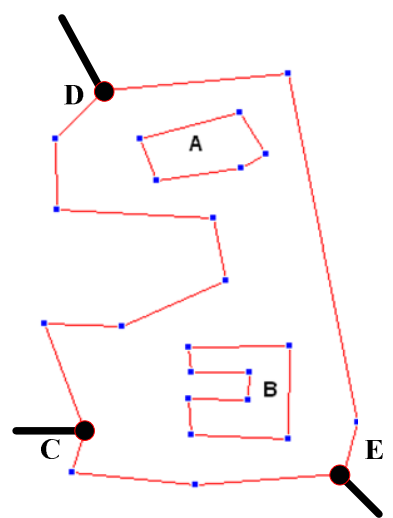

Figure 8 Area with islands

Due to these three orthogonal factors in characterizing shapes, we can model 12 general situations $(2 \times 2 \times 3)$. However, these characteristics of walking areas are not fixed but may vary. For example, some part of the boundary may be temporarily obstructed. In addition, environmental factors may affect if pedestrians wish to walk on grassy or other soft surfaces. Practical applications must consider these factors.

\section{Route PlanNing For Walking AReas}

For networks of pedestrian footpaths, sidewalks and building corridors, shortest path algorithms similar to those for road networks can be applied. However, when we have walking areas we must devise and integrate special algorithms to deal with the problems they create.

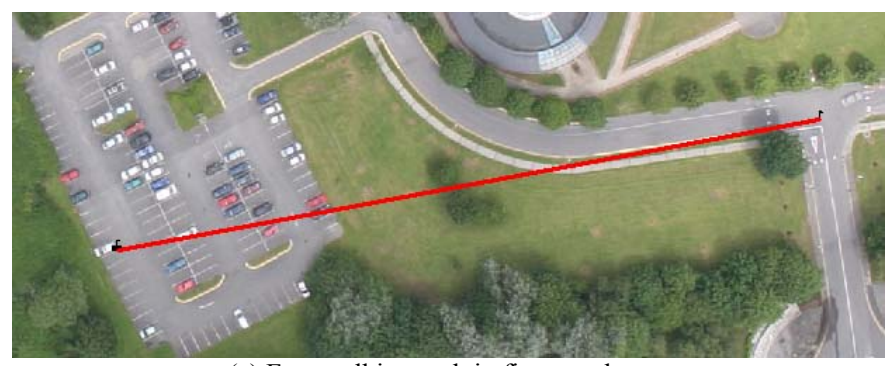

(a) Free walking path in fine weather

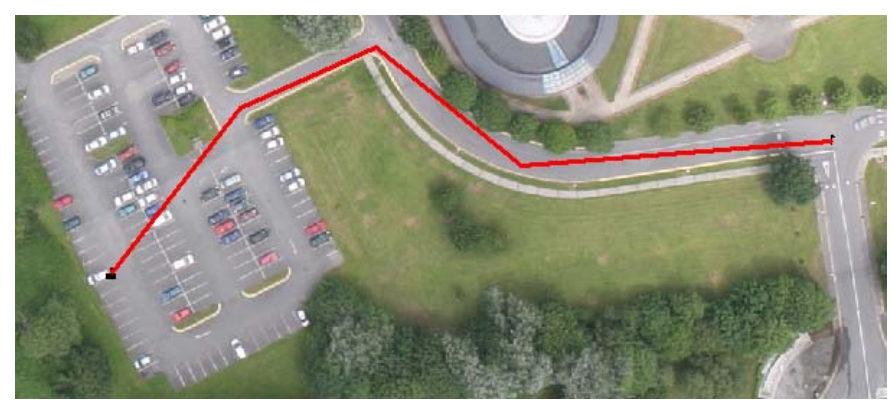

(b) Shortest path at night or on rainy days Figure 9 Various accessibility of walking area

\section{A. Pedestrian navigation through walking areas}

The three types of walking area characteristics mentioned in Section IV need to be considered when designing route planning algorithms and visualizing the results.

\section{- Walking area accessibility}

This is a service content/mode problem. The provider can make a menu to list different way-finding strategies for users to choose in various situations. The algorithms can be parameterized for different accessibility rules dpending on the mode.

- Concavo-convex characteristics of shapes

This characteristic affects the result considerably. Figure 12 shows the problem this may cause, especially in fixed entrance shapes with a concave boundary. The application should provide the shortest path within the walking area as shown.

\section{- numbers of islands in polygon}

If the walking area has island(s), the situation is quite similar to the second problem. The route must void the island(s).

\section{B. Research issues}

Traditionally (improved) Dijkstra's algorithm is effective in shortest path determination and outside walking areas is suitable. However, the inclusion of walking areas leads to a solution using two-level computing. One algorithm is for path planning outside walking areas. This is the first level.

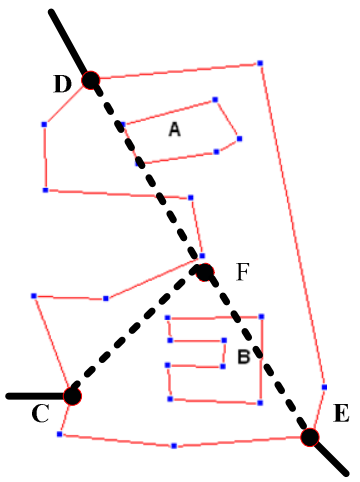

Fig.10 Simplified walking area as a link point for 1st level computing outside walking area

At this level, the walking area is regarded as one link point on the network, for example point $\mathrm{F}$ in Figure 10 could be used to represent the whole walking area. It could be the geometric center of the area, or its center of gravity. The dashed lines between $\mathrm{F}$ and pedestrian road links, which are the connections with the walking area, are newly generated pedestrian links. At this level, traditional path planning algorithms can be used to determine the optimal route.

The second level is used for inside walking areas. At this level, the computing focuses on the walking area and is more complex and different from that in the first level. There are three types of situation. 
1) Both the start and destination points are in different walking areas

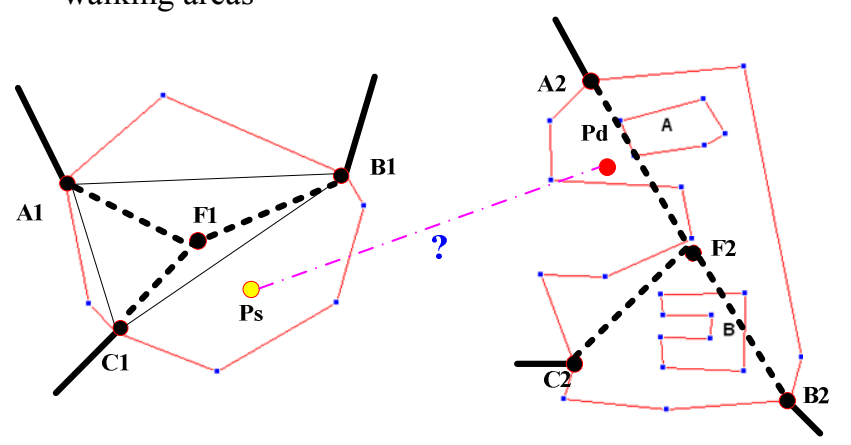

Fig.11 Start and destination points in different walking areas

Ps is the start point and $\mathrm{Pd}$ is the destination point. The mechanism to find the optimal route could be seen as following the typical procedure for fixed entrance type in Figure11.

It just uses the centre points of walking areas as pedestrian road nodes in the modified network. Links of $\mathrm{A} 1 \mathrm{~F} 1, \mathrm{C} 1 \mathrm{~F} 1, \mathrm{~B} 1 \mathrm{~F} 1, \mathrm{~A} 2 \mathrm{~F} 2, \mathrm{~B} 2 \mathrm{~F} 2$ and $\mathrm{C} 2 \mathrm{~F} 2$ also become part of the road network for optimal route computing. New or traditional optimal route planning algorithms could be adopted to obtain a suitable nodes string. As for the convex polygon, we could just use the real start and destination points to replace the ends of the nodes string. That is use the real point instead of the centre points.

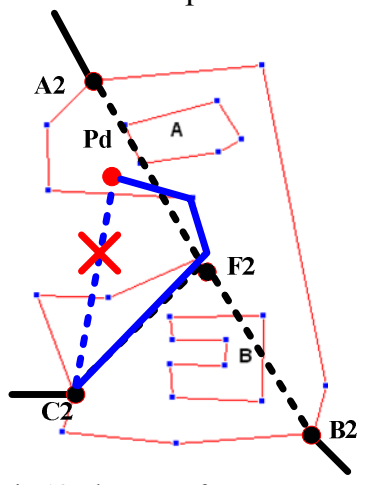

Fig.12 The case of concave areas

If it is a concave polygon, the algorithm should not provide a route crossing the boundary. The dashed route between $\mathrm{C} 2$ and $\mathrm{Pd}$ is not acceptable because for a fixed entrance shape, pedestrians usually cannot get through the boundary except at specific points. There are two constraints to be satisfied by a suitable route. One is it should be optimal. The other is that the route should be within the walking area. The proper result for shortest path is as the thick solid route in Figure 12. If it is a concave polygon and has some islands in it, another restriction should be added that is the route should not go through the islands.

2) One of the start and destination points is in a walking area

This type is a simpler version to that above. The analysis of above type is useful for this type also.
3) Both the start and destination points are in the same walking area

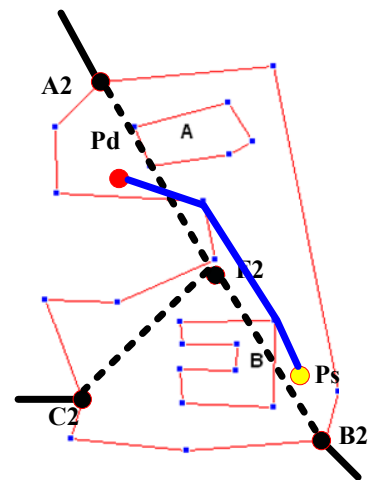

Fig.13 Both the start and destination points are in the same walking area

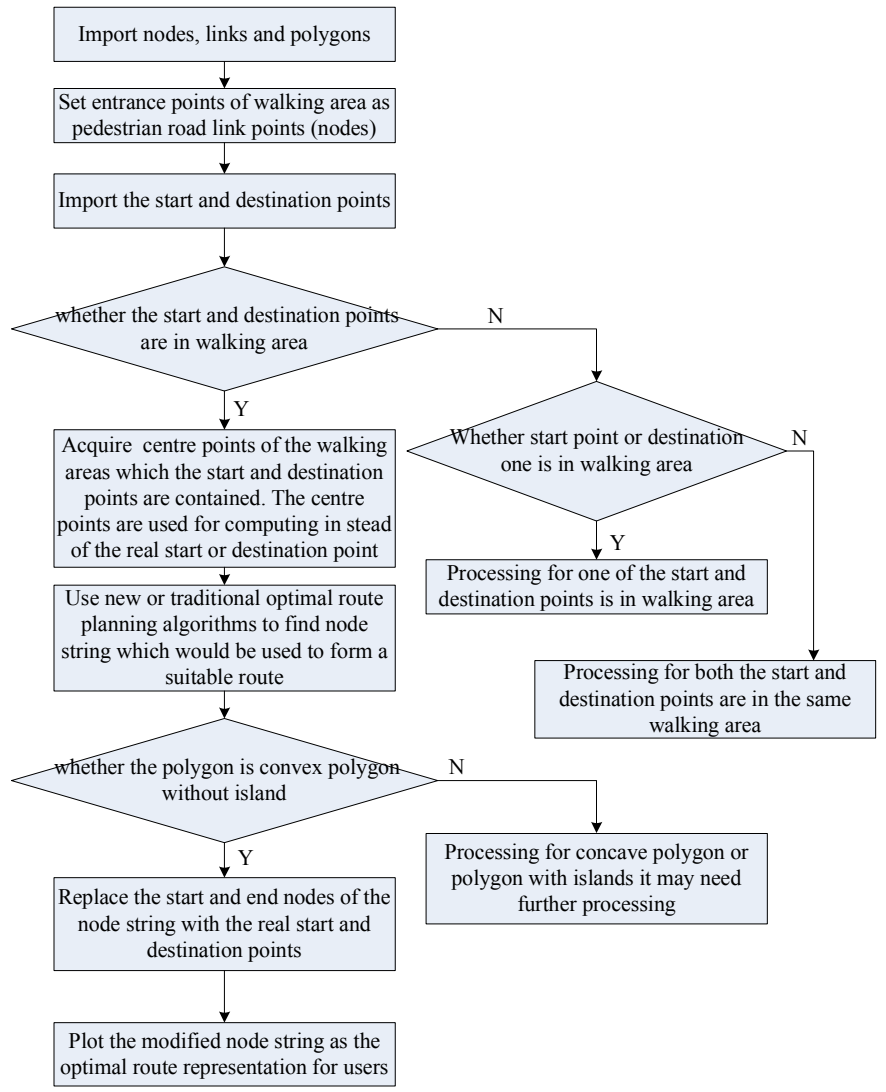

Fig14. processing Procedure of Route Planning

This type is a more complex than the second type. It should also use the mechanism put forward in analysis of the first type. It should also consider the concavo-convex characters of the polygon and need to judge whether the polygon has islands. The processing procedure could follow that in figure 14.

\section{CONCLUSIONS \& FUTURE WORK}

Most existing navigation services are designed for vehicles. For pedestrian navigation, we need to consider the special features of pedestrian path networks. This paper focused on one of these features, walking areas. It 
summarized that walking area accessibility, concavo-convex characteristics and the number of islands in polygons are the main factors to be considered in route planning and path visualization. Although general case studies were described, they still need to be developed in test applications for mobile terminals so user trials can be performed. Also, how to extend these navigation tasks to $3 \mathrm{D}$ scenarios (e.g. inside buildings) is also left for future work.

\section{ACKNOWLEDGMENT}

Research presented in this paper was funded by a Strategic Research Cluster grant (07/SRC/I1168) by Science Foundation Ireland under the National Development Plan. The authors gratefully acknowledge this support.

\section{REFERENCES}

[1] Dominique Bonte. The Mobile World Congress 2008: Pedestrian Navigation at Last, 11 Feb. 2008, [EB/OL]

http://www.abiresearch.com/Blog/Telematics_Blog/474

[2] Christian Gaisbauer and Andrew U.Frank. Wayfinding Model for Pedestrian Navigation. Proc. Of 11th AGILE International Conference on Geographic Information Science 2008.

[3] Jianghua Zheng, Jianwei Tao, Jianli Ding, Abudukim Abuliz, and Hanyu Xiang. Pedestrian Navigation Data Modelling for Hybrid Travel Patterns, Proceeding of Geoinformatics 2008. Proc. SPIE, Vol. 7144, 71442Y (2008)

[4] Masatoshi Arikawa, Shin'ichi Konomi and Keisuke Ohnishi. NAVITIME:Supporting Pedestrian Navigation in the Real World. PERVASIVE computing, Published by the IEEE Computer Society, July-September 2007, pp21-29

[5] Jianghua Zheng, YAN Lei, LIU Yuefeng, HE Lina. Study on Data Organization of Personal Navigation Services, Computer Engineering, 2006, 32(24):41-47 (in Chinese)

[6] Adam D.Sobek, Harvey J.Miller. U-Access:a web-based system for routing pedestrians of differing abilities. J Geograph Syst (2006)8: pp269-287

[7] Strothotte,T.,Fritz,S.,Michel,R.,Raab,A.,Petrie,H.,Johnson,V.,Reicher t,L.,Schalt, A.:Development of dialogue systems for a mobility aid for blind people:initial design and usability testing.In:Assets 1996:Proceedings of the second annual ACM conference on assistive technologies,pp.139-144.ACM Press,New York (1996)

[8] Ran,L.,Helal,S.,Moore,S.:Drishti:An Integrated Indoor/Outdoor Blind Navigation System and Service.In:Proc.of the Second IEEE International Conference on Pervasive Computing and Communications (PerCom 2004), pp.23-30. IEEE Press, New York (2004)

[9] Pressl,B.,Wieser,M.:A Computer-Based Navigation System Tailored to the Needs of Blind People. In: Miesenberger, K., Klaus, J., Zagler, W.,Karshmer,A.I.(eds.) ICCHP 2006.LNCS， vol.4061， pp.12801286. Springer, Heidelberg (2006)

[10] Bernhard Mayerhofer,Bettina Pressl,and Manfred Wieser. ODILIA-A Mobility Concept for the Visually Impaired, ICCHP 2008, LNCS 5105, pp1109-1116.

[11] Tetsuya MANABE,Takaaki HASEGAWA, Yoshihiro MATSUOKA, Seiji FURUKAWA, and Akira FUKUDA. On the M-CubITS Pedestrian WYSIWYAS Navigation Using Tile Carpets, Proceedings of the 2007 IEEE Intelligent Transportation Systems Conference, pp879-884, 2007

[12] Okan Yalak, Phillip Tomé, Valérie Renaudin. Hybridization of MEMS and Assisted GPS for Pedestrian Navigation, Inside GNSS , January/February 2007, pp34-42

[13] Ge'rard Lachapelle. Pedestrian navigation with high sensitivity GPS receivers and MEMS, Pers Ubiquit Comput(2007)11:pp481-488
[14] Masakatsu Kourogi,Nobuchika Sakata,Takashi Okuma,and Takeshi Kurata. Indoor/Outdoor Pedestrian Navigation with an Embedded GPS/RFID/Self-contained Sensor System, ICAT 2006,LNCS 4282,pp.1310-1321,2006.

[15] G.Retscher. Location Determination in Indoor Environments for Pedestrian Navigation, Proceeding of IEEE/ION PLAN 2006, pp547555,2006

[16] Joy Goodman,Phil Gray,Kartik Khammampad,and Stephen Brewster. Using Landmarks to Support Older People in Navigation, MobileHCI 2004, LNCS 3160,pp.38-48,2004.

[17] Tracy Ross, Andrew May,and Simon Thompson. The Use of Landmarks in Pedestrian Navigation Instructions and the Effects of Context, MobileHCI 2004,LNCS 3160,pp300-304, 2004

[18] Edgar-Philipp Stoffel, Bernhard Lorenz, and Hans Jurgen Ohlbach. Towards a Semantic Spatial Model for Pedestrian Indoor Navigation, ER Workshops 2007,LNCS 4802,pp.328-337,2007

[19] Antti Oulasvirta, Sara Estlander, Antti Nurminen. Embodied interaction with a $3 \mathrm{D}$ versus $2 \mathrm{D}$ mobile map, Pers Ubiquit Comput, 2008

[20] Seon-woo Lee and Kenji Mase. Recognition of walking behaviors for pedestrian navigation, Proceeding of the 2001 IEEE International Conference on Control Applications, 2001, pp1152-1155

[21] Kay Kitazawa And Michal Batty. Pedestrian Behaviour Modelling: An Application to Retail Movements using a Genetic Algorithm. Proceeding of DDSS 2004, [EB/OL]
[ www.casa.ucl.ac.uk/kay/paper/DDSS040703_e.pdf

[22] Zhan F B. Three Fastest Shortest Path Algorithms on Real Road Networks. Journal of Geographic Information and Decision Analysis, 1997, 1 (1): 69 82

[23] Pierre-Yves Gilliéron,Bertrand Merminod. Personal Navigation System For Indoor Applications, Proceeding of 11th IAIN World Congress October 21-24,2003,Berlin,Germany, pp1-15

[24] Shazia Haque, Lars Kulik, and Alexander Klippel. Algorithms for Reliable Navigation and Wayfinding, LNCS, Spatial Cognition V, LNAI 4387, pp. 308-326, 2007.

[25] Srihari Narasimhan, Hans-Joachim Bungartz. Methods for Optimal Pedestrian Task Scheduling and Routing.[EB/OL] http://www.cs.nott.ac.uk/ rxq/PlanSIG/narasimhan.pdf , 2006

[26] Lei Niu,Guobin Zhuo. An Improved Real 3d A*Algorithm For Difficult Path Finding Situation, Proceeding of the International Archives of the Photogrammetry,Remote Sensing and Spatial Information Sciences.Vol.XXXVII.Part B4.Beijing 2008

[27] Xin Zhang. Complete Route Planning Models and Algorithms for Public Transport Travelling[D]. Peking University, Beijing, 2007 